\title{
Estudo da Capacidade de Manter o Alerta em Pacientes com Fibromialgia por Meio do Teste da Manutenção da Vigília
}

\section{Degree of Daytime Sleepiness in Patients with Fibromyalgia through the Awakefulness Maintenance Test}

\author{
Djalma Gomes Ribeiro Sobrinho(1), Suely Roizenblatt(2), Antônio Carlos Lopes ${ }^{(3)}$, \\ Roberto Cordeiro A. Teixeira ${ }^{(1)}$, Sergio Tufik ${ }^{(2)}$
}

\begin{abstract}
RESUMO
Objetivo: Avaliar, de forma objetiva, a capacidade de manter o alerta em pacientes com fibromialgia por meio do teste da manutenção da vigília (TMV). Método: Foi realizado um estudo caso-controle de 15 pacientes com diagnóstico de fibromialgia com pelo menos $11 \mathrm{de}$ 18 pontos dolorosos e dor difusa nos últimos três meses. O grupocontrole foi constituído de 15 indivíduos hígidos pareados por idade e sexo, selecionados seqüencialmente. Os participantes responderam à escala de sonolência de Epworth e foram submetidos ao TMV. Foram realizadas quatro captações, cada uma com duração mínima de 20 minutos e com intervalo de duas horas. Considerou-se como normal a latência para o início do sono maior do que 20 minutos, e patológica a latência menor que 11 minutos. Resultados: $\mathrm{O}$ grupo com fibromialgia apresentou significante redução da latência para o início do sono no TMV, comparado ao grupo-controle 9,9 \pm 4,6 e $14,9 \pm 5,1$, respectivamente, $\mathrm{p}=0,01$, sendo que em $66,7 \%$ dos casos o resultado foi patológico, em comparação com $26,7 \%$ no grupocontrole $(\mathrm{p}=0,03)$. Não se observou correlação entre o resultado do TMV e a idade dos pacientes ou a pontuação na escala de sonolência de Epworth. Conclusão: Neste estudo-piloto, pacientes com fibromialgia apresentam redução da latência do sono no TMV.
\end{abstract}

Palavras-chave: fibromialgia, capacidade de alerta, sonolência excessiva diurna, teste de manutenção da vigília.

\section{INTRODUÇÃO}

A fibromialgia é uma síndrome dolorosa crônica que acomete $1 \%$ a $5 \%$ da população em geral $^{(1-3)}$. Caracteriza-se por queixa de dor musculoesquelética difusa e pela presença de pontos dolorosos em regiões anatomicamente determinadas. Na maioria dos casos, distúrbios do sono acompanham o quadro que se manifesta como sono fragmentado

\begin{abstract}
Objective: To assess in an objective way the degree of alertness in patients with fibromyalgia through the Maintenance Wakefulness Test (MWT). Method: Fifteen patients with fibromyalgia and 15 age and sex-matched healthy controls were sequentially selected. The inclusion criteria for fibromyalgia were the presence of at least 11 of the 18 tender points and diffuse pain during three months prior to evaluation. All participants answered the Epworth Sleepiness Scale (ESS) and underwent four 20 minutes sessions of MWT scheduled at 2-hour intervals. Mean sleep latency higher than 20 minutes or lower than 10 minutes was considered normal or pathological, respectively. Results: Although ESS scores did not differ between fibromyalgia patients and controls, the fibromyalgia group showed a significant decrease in the sleep latency by MWT ( $9.9 \pm 4.6$ and $14.9 \pm 5.1$, respectively, $p=0.01$ ). Pathological results were obtained in $66.7 \%$ of the patients, in comparison to $26.7 \%$ of the controls $(p=0.03)$. Conclusion: In this pilot study, patients with fibromyalgia showed decrease in the sleep latency by the Wakefulness Maintenance Test.
\end{abstract}

Keywords: fidromyalgia, daytime, excessive sleepiness, maintenance wakefulness test.

e não reparador ${ }^{(4)}$. A presença de despertares intermitentes e a intrusão de ondas alfa no sono não REM (rapid eye moviment) foram inicialmente descritas em $1975^{(5)}$. Estudo realizado em 2002 evidenciou que pacientes de fibromialgia, com queixa de sonolência diurna, tinham sintomatologia mais acentuada, inclusive maior número de pontos dolorosos ${ }^{(6)}$.

Recebido em 20/7/2007. Aprovado, após revisão, em 18/1/2008. Declaramos a inexistência de conflitos de interesse

Trabalho desenvolvido no Serviço de Neurofisiologia da Clínica Santa Juliana, pertencente à Fundação Educacional Jayme de Altavila (Fejal) e ao Setor de Neurofisiologia e Medicina do Sono do Hospital Geral Sanatório.

1. Professor da Faculdade de Medicina da Universidade Estadual de Ciências da Saúde de Alagoas (Uncisal), mestre pela Universidade Federal de São Paulo (Unifesp).

2. Professor(a) do Departamento de Psicobiologia da Escola Paulista de Medicina (EPM) da Unifesp.

3. Professor do Departamento de Medicina da Unifesp/EPM.

Endereço para correspondência: Djalma Gomes Ribeiro Sobrinho, Condomínio Aldebaran, Área Alfa, Lote C 24, Tabuleiro dos Martins, CEP 57080-900, Maceió, Al, e-mail: djalmaecmal@superig.com.br 
A sonolência diurna excessiva pode ser investigada por meio de questionários como o de Epworth ${ }^{(7)}$. No entanto, a avaliação tem maior acurácia quando são utilizados exames objetivos como o teste das múltiplas latências do sono (TMLS) e o teste da manutenção da vigília (TMV $)^{(8-14)}$. No entanto, deve-se considerar que fatores psicológicos podem influenciar as metodologias disponíveis para a avaliação da sonolência diurna, em especial fatores motivacionais e penalidades ${ }^{(14)}$.

Tendo como hipótese que distúrbios do sono na fibromialgia e a queixa de sono não reparador podem acarretar redução da capacidade de alerta, propõe-se este estudo no sentido de avaliar esse aspecto em pacientes com fibromialgia por meio do TMV.

\section{PACIENTES E MÉTODOS}

\section{CASUÍSTICA}

Foi realizado um estudo transversal e controlado de 15 pacientes com fibromialgia, e 15 indivíduos hígidos, selecionados seqüencialmente e pareados por idade e sexo. Os participantes foram encaminhados por reumatologistas ao Serviço de Neurofisiologia da Clínica Santa Juliana, pertencente à Fundação Educacional Jayme de Altavila (Fejal) e ao Setor de Neurofisiologia e Medicina do Sono do Hospital Geral Sanatório. Os critérios de inclusão foram: diagnóstico de fibromialgia ${ }^{(4)}$, idade entre 16 e 50 anos, presença de dor difusa ao menos nos últimos três meses, presença de pelo menos 11 dos 18 pontos dolorosos. Os critérios de exclusão foram: uso crônico de tabaco, patologias do sistema nervoso central, endócrinas, respiratórias ou cardiovasculares, uso de drogas que interfiram no padrão do sono e uso de álcool nos últimos quatro dias. A presente pesquisa foi aprovada pelo comitê de ética da Uncisal em 21 de dezembro de 2004 (processo $\mathrm{n}^{\circ} 288$ ). Todos os participantes assinaram o termo de consentimento livre e esclarecido.

\section{DESENHO DO ESTUDO}

Por meio de contato telefônico, os pacientes foram orientados a comparecer ao Serviço de Neurofisiologia da Clínica Santa Juliana e ao Hospital Geral Sanatório, com a carta de encaminhamento do reumatologista que incluiu a avaliação da presença de pelo menos 11 de 18 pontos dolorosos e a presença de dor difusa nos últimos três meses. Nessa ocasião, os participantes (pacientes e controles) responderam aos questionários no sentido de determinarem-se as manifestações da doença, a qualidade do sono nos últimos sete dias e a presença de sonolência diurna. O TMV foi realizado no dia seguinte, após o paciente ter tido seguramente uma noite de sono normal, constatada por meio de questionário.

Neste procedimento, avaliou-se a capacidade de permanecer acordado em ambiente silencioso, com temperatura adequada, luminosidade reduzida, e o paciente vestindo roupas confortáveis e permanecendo sentado. Duas horas após o voluntário ter despertado pela manhã, foram iniciadas as captações do registro eletrencefalográfico. Foram realizadas quatro captações com duração mínima de 20 minutos, com intervalo de duas horas entre elas. A quarta captação foi dispensada no caso de o voluntário ter dormido nos três primeiros testes.

\section{AVALIAÇÃo CLÍNICA}

Todos os participantes responderam a um questionário específico, que consistiu nos seguintes itens: duração e características da dor e da fadiga, tempo de diagnóstico da fibromialgia e tratamentos realizados.

\section{DIÁRIO DE SONO}

Todos os voluntários registraram as seguintes informações das sete noites que antecederam o exame: horário do despertar, tempo para iniciar o sono, número de despertares durante a noite e quanto tempo permaneceram acordados, quantas horas dormiram, números, ocasiões do dia e duração de cochilos, uso de álcool e cafeína, estresse e exercícios durante o dia, apetite ou perda deste durante o dia, como se sentiram durante o dia, qual o nível de irritação e uso de medicação. Foram também questionados o horário e o padrão de sono habituais e as características do sono na noite anterior ao TMV.

\section{ESCALA DE SONOLÊNCIA DE EPWORTH}

A escala de sonolência de Epworth é utilizada para a avaliação da sonolência diurna, consiste em oito questões com quatro possibilidades de resposta. É aplicada com $\mathrm{o}$ indivíduo respondendo com base em situações vividas anteriormente. Após a aplicação da escala, são somados os valores dados a cada situação e obtido um valor total, que quantificava a sonolência diurna. A escala apresenta um valor máximo de 24 pontos, sendo considerada sonolência diurna excessiva valores acima de 11 pontos $^{(7)}$.

\section{TESTE DA MANUTENÇÃo DA VIGÍLIA}

O exame foi realizado nos participantes que referiram padrão de sono habitual na noite anterior e que se abstiveram do uso de substâncias capazes de interferir no 
padrão do sono. O registro do eletrencefalograma (EEG) foi efetuado por eletrodos colocados de acordo com o sistema internacional 10-20 e mais outros para os seguintes canais: C3-A2, C4-Al, O1-A2 e O2-Al e ainda eletrodos para eletrooculograma (EOG) e eletromiografia (EMG) de mento.

No registro obtido, foi calculada a média das latências do sono e das latências do sono REM e realizada a análise do EEG. Foram avaliados o ritmo de base, as atividades superpostas na vigília, o padrão eletrencefalográfico e a possibilidade do surgimento de grafoelementos patológicos. Foi considerada normal a latência maior do que 20 minutos e a latência menor do que 11 minutos indicou redução na capacidade do alerta. A latência entre 11 e 20 minutos foi considerada resultado duvidoso ${ }^{(15)}$. O TMV é um ótimo método para estudar a capacidade de manter o alerta, porque verifica e quantifica, com métodos objetivos, a permanência do indivíduo acordado em condições que favoreçam o sono, como ambiente silencioso, com temperatura adequada, roupas e posição do corpo confortáveis.

\section{ESTATÍSTICA}

Os resultados foram apresentados em média \pm desviopadrão ou em porcentagem. Para os dados intervalares, foi utilizado o método $t$-student para amostras independentes e, para os dados nominais, o teste de Fisher. A análise da correlação entre dados foi efetuada pelo teste de correlação de Spearman. O nível de significância foi estabelecido em $5 \%$.

\section{RESULTADOS}

As características dos grupos controle e fibromialgia estão apresentadas na tabela 1 . Não se observou diferença entre os grupos no que se refere à idade ou à distribuição por gênero. No que se refere ao EEG de vigília, observouse ritmo alfa predominante em $100 \%$ no grupo-controle e em $86,7 \%$ no grupo com fibromialgia (Fisher, $\mathrm{p}=0,67$ ). A avaliação da capacidade do alerta está expressa na tabela 2. No grupo de voluntários com fibromialgia, evidenciou-se sonolência diurna por meio do questionário de Epworth, o que não ocorreu no grupo-controle, e o grupo com fibromialgia apresentou significante redução da latência para o início do sono no TMV. Não se observou correlação entre idade e o resultado do TMV e a escala de Epworth (Tabela 3). A porcentagem de indivíduos que apresentaram resultados normal, duvidoso e patológico, nos grupos controle e fibromialgia, está apresentada na tabela 4. Não se observou diferença significante entre as distribuições.
TABELA 1

CARACTERÍSTICAS DOS GRUPOS

\begin{tabular}{lcccc}
\hline & \multicolumn{2}{c}{ Grupos } & \multicolumn{2}{c}{ Estatística } \\
\cline { 2 - 5 } & Controle & Fibromialgia & t-value & $\mathbf{p}$ \\
\hline Idade & $44,7 \pm 9,2$ & $51,3 \pm 9,3$ & $-2,0$ & 0,06 \\
F/M & $14 / 1$ & $14 / 1$ & & 1,00 \\
\hline
\end{tabular}

Teste $t$ para amostras independentes e teste de Fisher $\mathrm{F} / \mathrm{M}=$ gênero feminino/masculino

TABELA 2

AvaliaÇ̃̃o DA Vigília

\begin{tabular}{lcccc}
\hline & \multicolumn{2}{c}{ Grupos } & \multicolumn{2}{c}{ Estatística } \\
\cline { 2 - 5 } & Controle & Fibromialgia & t-value & $\mathbf{p}$ \\
\hline Epworth & $7,2 \pm 4,3$ & $10,3 \pm 6,1$ & $-1,5$ & 0,14 \\
TMV & $14,9 \pm 5,1$ & $9,9 \pm 4,6$ & 2,8 & 0,01 \\
\hline
\end{tabular}

Teste $t$ para amostras independentes

TMV = teste de manutenção da vigília

TABELA 3

CorrelaÇões ENTRE OS PARÂMETROS ESTUdAdOS $\left(\mathrm{R}_{\mathrm{S}}\right)$

\begin{tabular}{lcc}
\hline & TMV & Epworth \\
\hline Idade & $-0,34$ & 0,17 \\
TMV & & $-0,26$ \\
\hline
\end{tabular}

Teste de Spearman

TMV = teste de manutenção da vigília

TABELA 4

TESTE DE MANUTENÇÃO DA VIGÍlLIA

\begin{tabular}{lcc}
\hline & Controle $(\mathrm{n}=15)$ & Fibromialgia $(\mathrm{n}=15)$ \\
\hline Normal & $6(40 \%)$ & $1(6,7 \%)$ \\
Duvidoso & $5(33,3 \%)$ & $4(26,7 \%)$ \\
Patológico & $4(26,7 \%)$ & $10(66,7 \%)$ \\
\hline
\end{tabular}

\section{DISCUSSÃO}

A escassez de pesquisas objetivas sobre o estado de vigília em pacientes com fibromialgia motivou esse estudo uma vez que alterações estruturais e a queixa de sono não reparador ${ }^{(5)}$ poderiam acarretar sonolência diurna excessiva, como ocorre em outras patologias associadas à fragmentação do sono ${ }^{(16-17)}$.

No sentido de diferenciar a sonolência diurna excessiva da fadiga, a qual corresponde a um sintoma muscular, também freqüentemente referido pelos pacientes, alguns autores, 
utilizando a escala de Epworth, evidenciaram a presença de sonolência diurna excessiva na fibromialgia e correlacionaram tal achado com a intensidade das queixas ${ }^{(6)}$.

Todavia na investigação da sonolência diurna excessiva, a avaliação é feita com mais acurácia quando são utilizados métodos objetivos como o TMV e o TMLS. A pesquisa por meio de questionários, como a escala de Epworth, depende exclusivamente das informações dos indivíduos, que podem ter percepção inadequada do sono ${ }^{(18)}$.

Estudos desenvolvidos têm relatado discrepâncias entre os resultados obtidos com o TMLS e a escala de Epworth $^{(7,19)}$. Os métodos objetivos são melhores do que a escala de Epworth na avaliação da sonolência diurna em pacientes com síndrome da apnéia obstrutiva do sono ${ }^{(12)}$. Investigando a hiperssonia em indivíduos com narcolepsia, observou-se que a escala de Epworth não media tão bem os níveis de sonolência como o $\mathrm{TMV}^{(20)}$.

O TMV mensura a habilidade para permanecer acordado, e o TMLS avalia a rapidez para dormir em uma situação soporífera e ao aparecimento de sono REM precoce (essencial para o diagnóstico de narcolepsia). Isso pode explicar por que a correlação entre os dois testes não é alta ${ }^{(21)}$. Embora o TMV reflita melhor a sonolência excessiva após alguma intervenção, esse método foi utilizado no presente estudo, cujo objetivo foi avaliar a capacidade do alerta ${ }^{(10)}$.

Comparando-se os resultados obtidos entre o grupo controle e o de portadores de fibromialgia (Tabela 2), o TMV

\section{REFERÊNCIAS}

1. Wolfe TA, Ross K, Anderson J, Russell J: Aspects of fibromyalgia in the general population: Sex, pain threshold, and fibromyalgia symptoms. J Rheumatol 22: 151-6, 1995.

2. White KP, Speechley M, Harth M, Ostbye T: The London Fibromyalgia epidemiology study: comparing teh demographic and clinical characteristics in 100 random community cases of fibromyalgia versus controls. J Rheumatol 26: 1577-85, 1999.

3. Kato K, Sullivan PF, Evengard B, Pedersen NL: Chronic widespread pain and its comorbidities: a population-based study. Arch Intern Med 166: 1649-54, 2006.

4. Wolfe F, Smythe HA, Yunus MB, et al.: The American College of Rheumatology 1990 criteria for the classification of fibromyalgia: report of the multicenter criteria committee. Arthritis Rheum 33: 160-72, 1990.

5. Moldofsky H, Scarisbrick P, England R, Smythe H: Musculoskeletal symptoms and non REM sleep disturbance in patients with fibrositis syndrome and healthy subjects. Psychosom Med 537: 341-51, 1975.

6. Sarzi-Puttini P, Rizzi M, Andreoli A, et al.: Hypersomnolence in fibromyalgia syndrome. Clin Exp Rheumatol 20: 69-72, 2002. evidenciou que efetivamente os portadores de fibromialgia tinham diminuição da latência do sono, indicando menor capacidade de alerta e, indiretamente, sonolência diurna patológica. A tabela 4 revela que $66,7 \%$ dos indivíduos do grupo fibromialgia apresentam menor capacidade de alerta, e que em $26,7 \%$ do grupo controle notou-se o transtorno.

No que se refere à amostra pesquisada, o presente estudo tem como limitação a tendência do grupo-controle ser mais jovem, embora a diferença da faixa etária não é significante. Deve-se considerar que a sonolência diurna excessiva é um problema freqüente, causado por diversos fatores como insuficiência voluntária de sono, transtornos do sono como síndrome da apnéia obstrutiva do sono, síndrome das pernas inquietas e narcolepsia. Tais aspectos não foram investigados nos pacientes com valor patológico obtido no TMV, o que justifica a necessidade de se desenvolver estudos mais abrangentes que envolvam maior número de participantes e que incluam a polissonografia em sua metodologia. Consideramos ainda que, apesar de wash-out de quatro dias, pacientes em uso de tricíclicos podem estar com exames alterados não pela fibromialgia, e sim pela medicação. Assim, este pode ser considerado um estudo-piloto.

\section{CONCLUSÃO}

Neste estudo-piloto, pacientes com fibromialgia apresentam redução da latência do sono no TMV.

7. Johns MW: A new method for measuring daytime sleepiness: The Epworth Sleepiness Scale. Sleep 14: 540-5, 1991.

8. Merrill M, Mitler MA, Hirshkowitz CM: Evaluating Sleepiness. Principles and Practice of Sleep Medicine. 2.ed. Philadelphia: Saunders; 2006. p. 1.

9. Sangal RB, Mitler MM, Sangal JM: Subjective sleepiness ratings (Epworth sleepiness scale) do not reflect the same parameter of sleepiness as objective sleepiness (maintenance of wakefulness test) in patients with narcolepsy. Neurophysiol 110(12): 2131-5, 1999.

10. Bittencourt LRA, Santos RS, Santos RF, Pires MLN, Mello MT: Sonolência Excessiva. Rev Bras Psiquiatria 27: 16-21, 2005.

11. Dauvilliers Y, Buguet A: Hypersomnia. Dialogues Clin Neurosci 7(4): 347-56, 2005.

12. Fong SYY, Ho CKW, Wing YK: Comparing MSLT and ESS in the measurement of excessive daytime sleepiness in obstructive sleep apnoea syndrome. J Psychosom Res 58: 55-60, 2005.

13. Littner MR, Kushida C, Wise M, et al.: Standards of Practice Committee of the American Academy of Sleep Medicine. Practice parameters for clinical use of the multiple sleep latency test and the maintenance of wakefulness test. Sleep 28: 113-21, 2005. 
14. Wise MS: Objective measures of sleepiness and wakefulness: application to the real world? J Clin Neurophysiol 23: 39-49, 2006.

15. Doghramji K, Mitler MM, Sangal RB, et al.: A normative study of the Maintenance of Wakefulness Test (MWT). Electroencephalogr Clin Neurophysiol 103: 554-62, 1997.

16. Bonnet MH.: Performance and sleepiness as a function of frequency and placement of sleep disruption. Psychophysiology 23: 263-71, 1986.

17. Martin SE, Wraith PK, Deary IJ, Douglas NJ: The effect of nonvisible sleep fragmentation on daytime function. Am J Respir Crit Care Med 155: 1596-601, 1997.
18. Olson LG, Cole MF, Ambrogetti A: Correlations among Epworth Sleepiness Scale scores, multiple eleep latency tests and psychological symptoms. J Sleep Res 7: 248-53, 1998.

19. Benbadis SR, Mascha E, Perry MC, Wolgamuth BR, Smolley LA, Dinner DS: Association of the Epworth Sleepiness Scale and the multiple sleep latency test in a clinical population Ann Intern Med 30: 289-92, 1999.

20. Sangal RB, Mitler MM, Sangal JM: Subjective sleepiness ratings (Epworth sleepiness scale) do not reflect the same parameter of sleepiness as objective sleepiness (maintenance of wakefulness test) in patients with narcolepsy. Neurophysiol 110(12): 2131-5, 1999.

21. Sangal RB, Thomas L, Mitler MM: Maintenance of wakefulness test and multiple sleep latency test. Measurement of different abilities in patients with sleep disorders. Chest 101: 898-902, 1992. 